\title{
A neural network based UHE neutrino reconstruction method for the Askaryan Radio Array (ARA)
}

\section{Yue Pan* on behalf of the ARA Collaboration}

(a complete list of authors can be found at the end of the proceedings)

University of Delaware,

Newark, DE, USA

E-mail: yuepan@udel.edu

The Askaryan Radio Array (ARA) is an ultra-high energy (UHE) neutrino $\left(E_{v}>10^{17} \mathrm{eV}\right)$ detector at South Pole. ARA aims to utilize radio signals detected from UHE neutrino interactions in the glacial ice to infer properties about the interaction vertex as well as the incident neutrino. To retrieve these properties from experiment data, the first step is to extract timing, amplitude and frequency information from waveforms of different antennas buried in the deep ice. These features can then be utilized in a neural network to reconstruct the neutrino interaction vertex position, incoming neutrino direction and shower energy. So far, vertex can be reconstructed through interferometry while neutrino reconstruction is still under investigation. Here I will present a solution based on multi-task deep neural networks which can perform reconstruction of both vertex and incoming neutrinos with a reasonable precision. After training, this solution is capable of rapid reconstructions (e.g. $0.1 \mathrm{~ms} /$ event compared to $10000 \mathrm{~ms} / \mathrm{event}$ in a conventional routine) useful for trigger and filter decisions, and can be easily generalized to different station configurations for both design and analysis purposes.

$37^{\text {th }}$ International Cosmic Ray Conference (ICRC 2021)

July 12th-23rd, 2021

Online - Berlin, Germany

\footnotetext{
*Presenter
} 


\section{Introduction}

The Askaryan Radio Array (ARA) is an UHE neutrino detector currently under construction at South Pole. This proceeding describes a multi-task convolutional neural network $(\mathrm{CNN})$ trained on simulated data to provide rapid and accurate reconstructions in the case of candidate neutrino events. The CNN takes extracted event features as input and outputs an estimate of the incident neutrino direction and the position of the interaction vertex in the south pole ice.

As of 2021, ARA includes 5 stations. An ARA station has 4 strings each with 4 measurement antennas. The 16 antennas form a rectangular solid with sides of $15 \times 15 \times 20 \mathrm{~m}$ as shown in Fig. 1 . To measure polarization of incoming signals, 8 of the antennas are vertically polarized (VPol) and the other 8 are horizontally polarized (HPol), mounted alternatively on each string. Details of the station configuration and performance can be found in [1] [2].

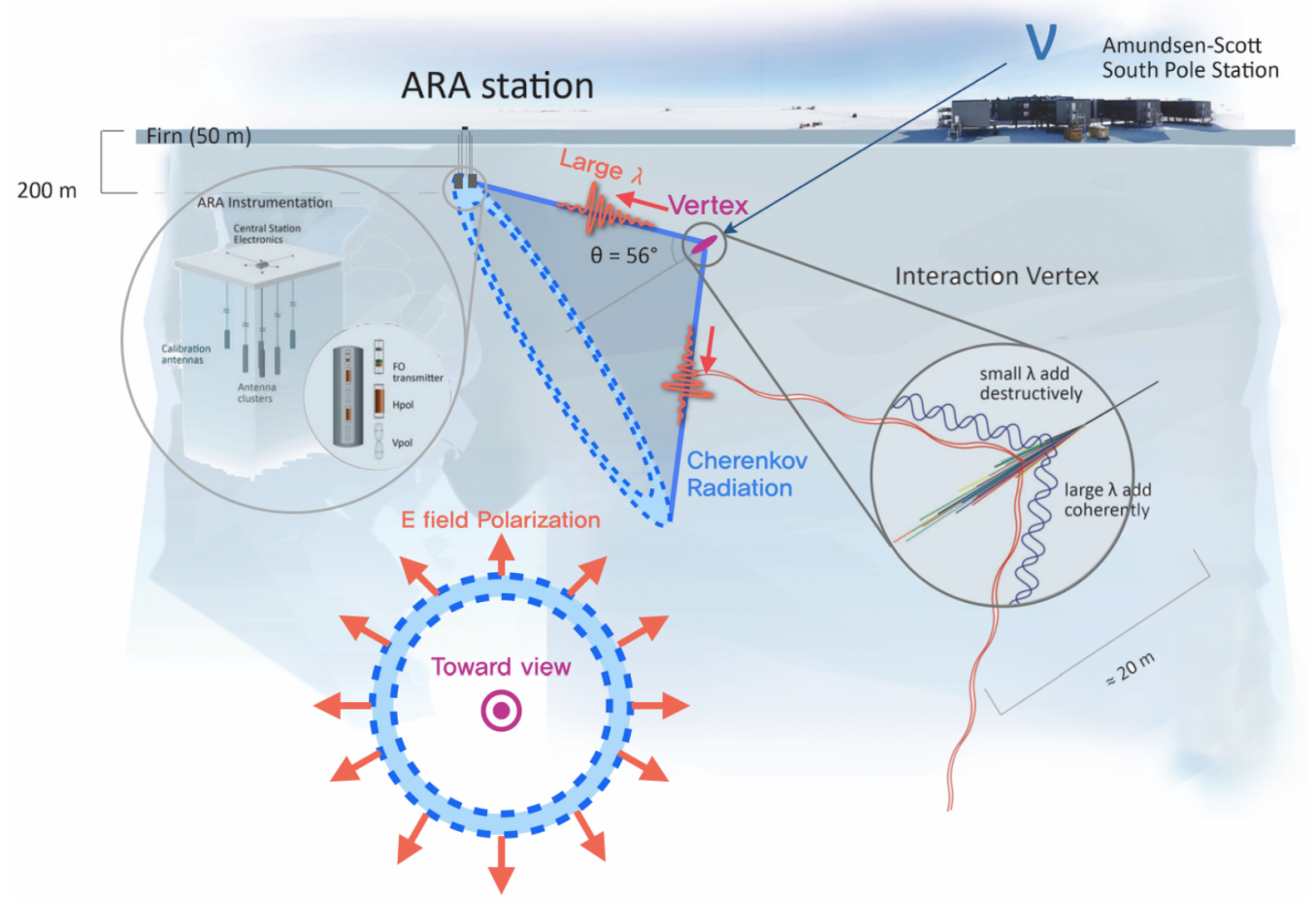

Figure 1: Neutrino radio detection by an ARA station.

When a neutrino interacts in ice, a cascade of electrons and positrons is created. This relativistic "spark" generates a radio frequency pulse via the Askaryan effect [3]. Due to the low temperature in the ice, the attenuation length of the radio signals is of order of $1 \mathrm{~km}$, which allows the detector to be deployed sparsely [4]. Each ARA station is designed to detect these signals from a volume of order a hundred $\mathrm{km}^{3}$ of ice, which provides an aperture of $10^{4} \mathrm{~m}^{2} \mathrm{sr}$ at $10^{18.5} \mathrm{eV}$ [2].

To reconstruct a neutrino vertex in a conventional way, relative timing of signals from antennas at different locations is used to reconstruct the arrival direction of rays, which can be traced back to find the interaction vertex. Relative amplitudes from pairs of polarized antennas are used to reconstruct the polarization of the incident electric field, which is used to resolve the direction of the ray relative to the shower axis and, in turn, the neutrino momentum. 
The data set used in this proceeding is generated with a Monte Carlo simulation tool for UHE neutrino experiments, NuRadioMC [5] [6]. It can simulate the full chain from Askaryan radio signal generation all the way to recording of waveforms by a user defined detector. NuRadioMC is a python-steered, modular framework for general detector configurations, e.g. it can simulate and be used to study a variety of future detector designs. In this proceeding, A2 configuration is used in simulation to generate the data set.

The proceeding first describes how the data set is produced and processed to meet the requirement of a convolutional neural network. The choice of some network features, e.g. its architecture, input and output structure, loss functions is then justified in Sec. 3. The reconstruction results and some performance measurements are illustrated in Sec. 4. Finally, a summary and some future works conclude the proceeding.

\section{Data}

The data set is generated with NuRadioMC. Simulations are produced run by run by throwing $100 \mathrm{k}$ events with an energy spectrum ranging from $10^{16.5} \mathrm{eV}$ to $10^{20} \mathrm{eV}$ with a step of $10^{0.5}$. The thrown events in each run are distributed evenly in a cylinder around the station center, while the directions of neutrinos are isotropic in azimuth and $\cos$ (zenith). Depending on the specific station design, the distribution of the vertex locations and the neutrino direction may vary. With the A2 configuration, the distribution of simulated events at $10^{18.5} \mathrm{eV}$ is shown in Fig. 2. Fig. 2a shows that triggered events are symmetrically distributed in azimuth and within a certain distance due to ice attenuation. There is a shadow line in Fig. $2 \mathrm{~b}$ above which no event can illuminate the detector due to refraction in the ice. Fig. $2 c$ shows that only those neutrinos coming in a specific zenith range can trigger the station. Neutrinos coming from below, i.e. those with zenith angles greater than $100 \mathrm{deg}$, are absorbed by the Earth, while those down-going, i.e. with zenith less than $50 \mathrm{deg}$ cannot radiate towards the detector due to the restriction of the Cherenkov angle [3].

A typical set of waveforms with noise is in Fig. 3. Each antenna records either 0 or 2 pulses from the simulation [7]. In the case of 2 pulses, the first is called the direct pulse (D) and the second the refracted/reflected pulse (R). To extract timing and amplitude information, a $31.25 \mathrm{~ns}$ window around each pulse is taken according to true simulation information at this stage, and a corresponding Hilbert envelope is calculated. An integral of the envelope within each window is used as amplitude information. The $+/$ - sign of the polarized electric field is supplied as a feature to improve reconstruction of the incident neutrino direction. To get timing, channel $0 \mathrm{D}$ pulse is chosen as the reference, and is assigned $0 \mathrm{~ns}$ timing. The relative signal arrival time from all other pulses is then calculated with respect to the reference waveform by a cross-correlation technique.

With a full data set of about $274 \mathrm{k}$ events in 320 runs, those events with missing signals in any of the channels are excluded, which happens mostly at the boundary of the vertex phase space. After cleaning, a remaining $243 \mathrm{k}$ events suggests an efficiency of $89 \%$ of the events with discernible D and $\mathrm{R}$ pulses. The cleaned data set is then split into $90 \%, 5 \%, 5 \%$ for training, validation and test respectively. A scaling of the input features by subtracting the training mean and dividing by the standard deviation is applied to all three sets before providing the data to the neural network so that all the features are of the same order and are weighted by their significance. 


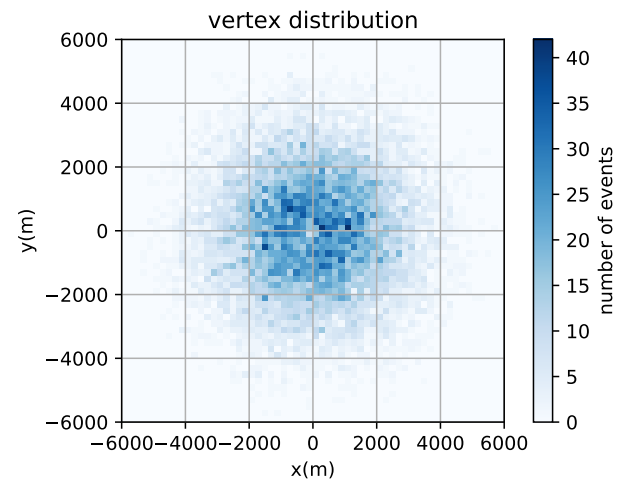

(a)

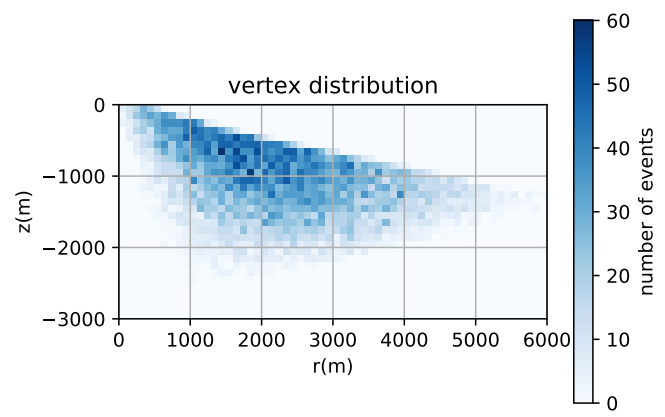

(b)

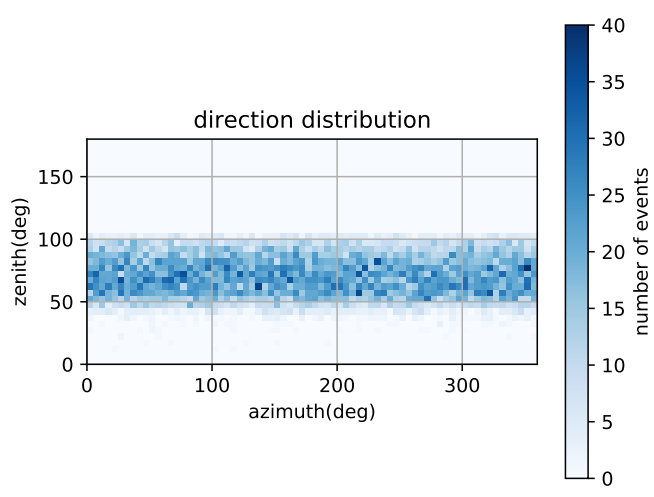

(c)

Figure 2: Triggered events from a simulation of A2 by NuRadioMC. The events are at $10^{18.5} \mathrm{eV}$.

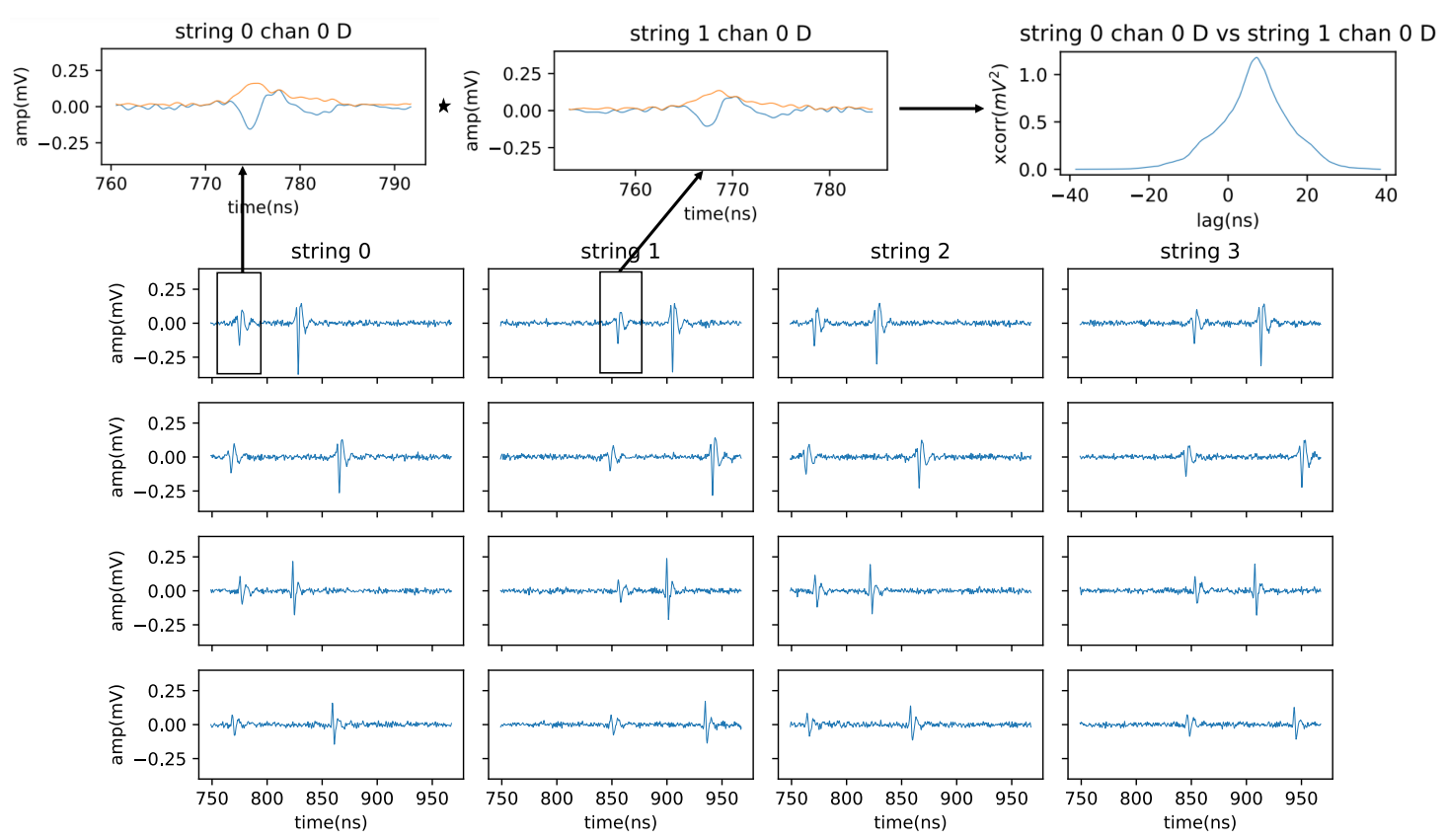

Figure 3: Waveforms from a $10^{18.5} \mathrm{eV}$ neutrino event at $1768 \mathrm{~m}$ from the detector. 


\section{Method}

In this proceeding, a multi-task convolutional neural network is developed to reconstruct neutrino vertex and direction. The architecture of the network is inspired by the work in IceCube [8] and is shown in Fig. 4. The first part consists of convolutional layers with $3 \times 3$ kernels to learn hidden characteristics and handle the high dimensional nature of the input features. The total number and the number of filters of the convolutional layers are hyper-parameters that can be tuned to improve the performance. The second half includes only dense layers after flattening the $3 \mathrm{~d}$ intermediate output from the first part, of which the total number and number of nodes are hyper-parameters as well. The input layer is a $(4,4,6)$ matrix to better reflect the detector configuration. The output is a vector of length 7 to predict both neutrino vertex and neutrino direction in the same model.

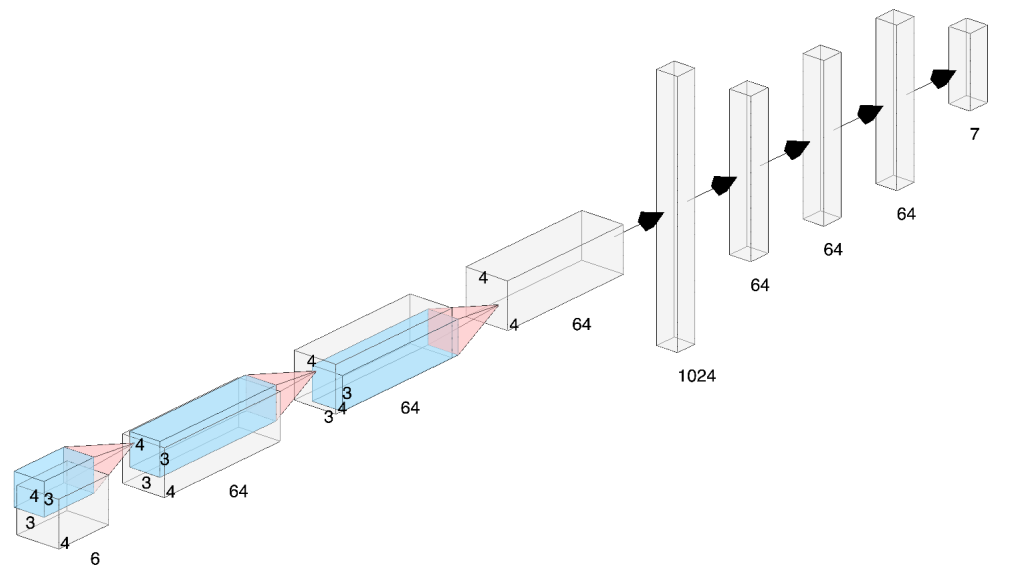

Figure 4: A diagram of the optimized network architecture.

According to the discussion in Sec. 1, the relevant input features are timing, amplitude and ratio of amplitudes from HPol and VPol antenna pairs. The features are aligned as shown in Fig. 5. For each type of feature, they are aligned string by string in column direction with VPol antennas in the top two rows and HPol antennas in the bottom two. Different types of features are aligned along the channel axis in a sequence of timing from $\mathrm{D}$ pulses, timing from $\mathrm{R}$ pulses, amplitude from D pulses, amplitude from R pulses, ratio of corresponding VPol and HPol from D pulses, ratio of corresponding VPol and $\mathrm{HPol}$ from $\mathrm{R}$ pulses. In addition, a periodic padding is applied by inserting string 3 features ahead of string 0 and appending string 0 features to string 3 to account for azimuth continuity.

The outputs are the measurements of neutrino vertex and direction, which is described in Tab. 1. Mean squared percentage error (MSPE) loss MSPE $=\frac{1}{n} \sum_{i=1}^{n}\left(\left(y_{\text {true }, i}-y_{\text {pred }, i}\right) / y_{\text {true }, i}\right)^{2}$ is used for $r$ to give similar training weights to near and far events. Mean squared error (MSE) loss $M S E=\frac{1}{n} \sum_{i=1}^{n}\left(y_{\text {true }, i}-y_{\text {pred }, i}\right)^{2}$ is applied to all the angular outputs since the uncertainty in a vector should not depend on the direction of the vector. The application of trigonometric functions to azimuth angles of both vertex and direction is to avoid the discontinuity at $0 / 360$ deg caused by the MSE loss. 


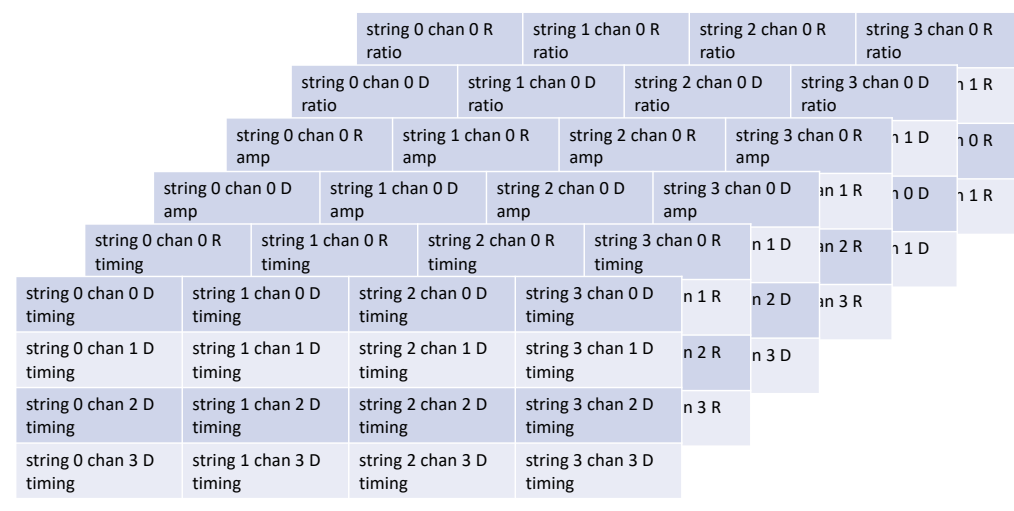

Figure 5: The input feature structure.

\begin{tabular}{|c|c|c|}
\hline Label & Explanation & Loss \\
\hline$r$ & Horizontal distance from the station center to a vertex & MSPE \\
\hline $\sin \alpha, \cos \alpha$ & Azimuth angle from the station center to a vertex & MSE \\
\hline$\beta$ & Zenith angle from the station center to a vertex & MSE \\
\hline $\sin \phi, \cos \phi$ & Azimuth angle pointing back to where a neutrino comes from & MSE \\
\hline$\theta$ & Zenith angle pointing back to where a neutrino comes from & MSE \\
\hline
\end{tabular}

Table 1: A description of outputs.

\section{Results}

After training a neural network, its performance is estimated by applying it to the 5\% test data set. The test set is never used in training, so it is a good source to check the model performance on unseen data. The error distributions are shown in Fig. 6. $r$ is measured in relative error as $\Delta r / r=$ $\left(r_{\text {pred }}-r_{\text {true }}\right) / r_{\text {true }}$, while all the angular outputs are measured in error, e.g. $\Delta \beta=\beta_{\text {pred }}-\beta_{\text {true }}$. All the distributions are normalized to 1 . The width of an error distribution $\sigma$ is calculated as half of the difference between the 84 percentile and the 16 percentile to measure the reconstruction accuracy of the middle $68 \%$ events. The median of the error distribution $m$ is the 50 percentile.

All the distributions are centered around zero, suggesting there is no systematic offset between predicted and true values. $\sigma_{r}$ is well below $10 \%, \sigma_{\beta}$ and $\sigma_{\alpha}$ are within $0.5 \mathrm{deg}$. The use of true information for timing seed and polarity requires further investigation. The neutrino direction reconstruction has wider error distributions with $\sigma_{\theta}$ of $4 \mathrm{deg}$ and $\sigma_{\phi}$ of $6 \mathrm{deg}$. These may be correlated, depending on the orientation of the Cherenkov cone. On the other hand, a small scale detector like A2 relies on frequency to determine the launch direction relative to the Cherenkov cone, so lack of frequency information as inputs may further degrade the performance.

In addition to the test on unseen data to assess generalization, the model stability is estimated by applying 10 fold cross validation. The results of $m$ and $\sigma$ of all the outputs with error bars are in Fig. 7. $m_{r}, m_{\beta}, m_{\alpha}, m_{\phi}$ 's going across zero within one standard deviation shows there's no systematic bias. $m_{\theta}$ is $0.1 \mathrm{deg}$ above zero. Although the error bars for $\sigma_{\theta}$ and $\sigma_{\phi}$ are wider than those of $\sigma_{r}, \sigma_{\beta}$ and $\sigma_{\alpha}$, the width of all the error bars in Fig. 7b are small, suggesting the model is 


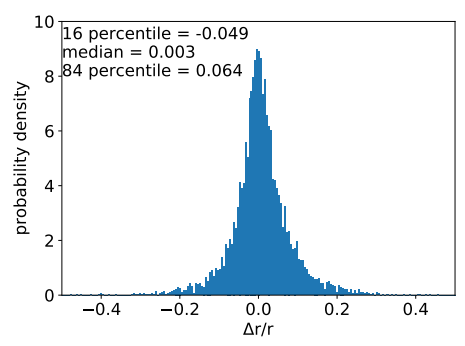

(a)

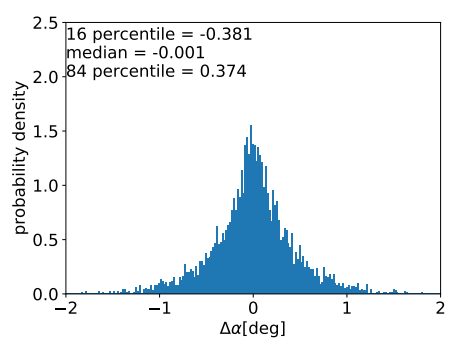

(b)



(c)



(d)

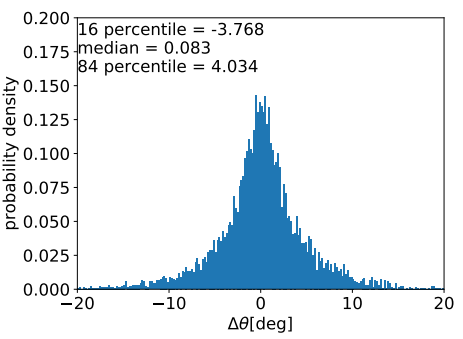

(e)

Figure 6: The error distribution.

stable in terms of training and testing on different data sets.

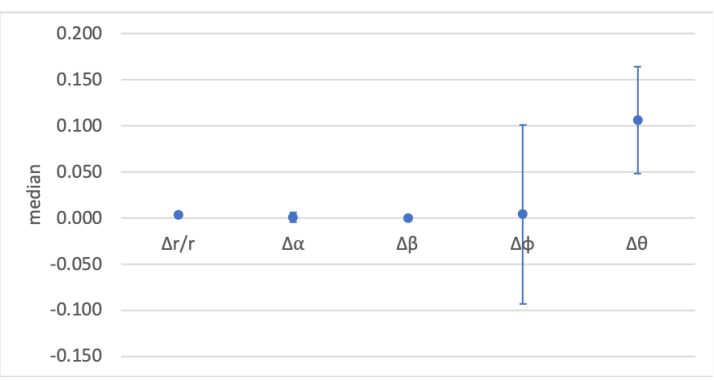

(a)

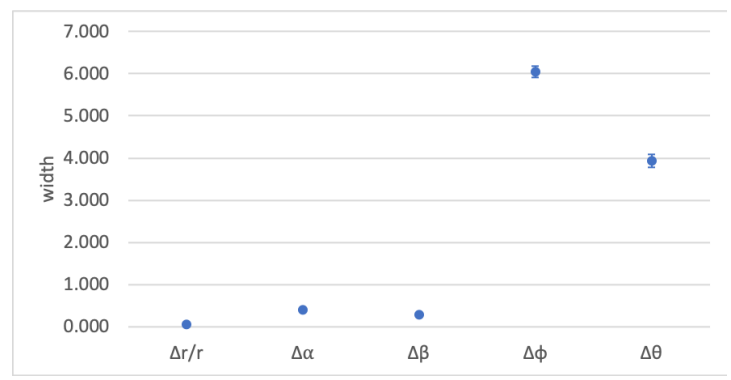

(b)

Figure 7: Median $m$ and width $\sigma$ from 10 fold cross validation.

The relationship between data size and performance is also studied for the purpose of estimating the performance limit. The whole data set contains 320 runs. A set of network models is trained with 40, 80, 160 and 320 runs and the performance is estimated. The results in Fig. 8 are normalized according to the performance of the model with 40 runs. According to the figures, having more data can potentially lower $\sigma$.

\section{Summary and Outlook}

As illustrated herein, the multi-task convolutional neural network can give a stable and precise reconstruction results for both neutrino vertex and neutrino direction for a majority of triggered neutrino events. However, there are still some future works to do. First, the method of extracting 


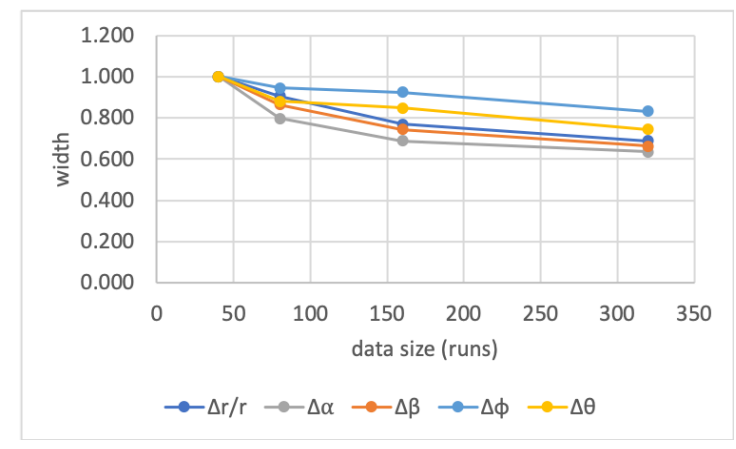

Figure 8: Width $\sigma$ from a set of models trained with 40, 80, 160 and 320 runs.

timing and amplitude features from waveforms can be replaced by another neural network, which can then be connected to the reconstruction network to make an end-to-end model. In that case, the model won't rely on simulated true information about the pulse window and polarity. Quality cuts can then be applied to keep only high SNR events, which can lower the efficiency illustrated in Sec. 2. Second, frequency features should be investigated to reconstruct shower energies. Third, due to the flexibility of a neural network model, it can be generalized to other station configurations to study reconstruction, e.g. IceCube-Gen2 [9]. Finally, the model should be applied to real data set for test. One possibility is to apply the trained network to SpiceCore data that was taken in 2018.

\section{References}

[1] ARA Collaboration, P. Allison et al. Phys. Rev. D 93 (2016) 082003.

[2] ARA Collaboration, P. Allison et al. Phys. Rev. D 102 (2020) 043021.

[3] G. A. Askar'yan Soviet Journal of Experimental and Theoretical Physics 21 (1965) 658.

[4] S. Barwick, D. Besson, P. Gorham, and D. Saltzberg Journal of Glaciology 51 (2005) 231-238.

[5] C. Glaser, D. Garcia-Fernandez, A. Nelles, et al. Eur. Phys. J. C80 (2020) 77.

[6] C. Glaser, A. Nelles, I. Plaisier, C. Welling, et al. Eur. Phys. J. C79 (2019) 464.

[7] ARA Collaboration, J. Kelley, M.-Y. Lu, D. Seckel, Y. Pan, and D. Z. Besson PoS ICRC2017 (2018) 1030.

[8] IceCube Collaboration, R. Abbasi et al. (2021) .

[9] IceCube Collaboration, M. G. Aartsen et al. J.Phys. G 48 no. 6, (2021) 060501. 


\section{Full Author List: ARA Collaboration}

P. Allison ${ }^{1}$, S. Archambault ${ }^{2}$, J.J. Beatty ${ }^{1}$, M. Beheler-Amass ${ }^{3}$, D.Z. Besson ${ }^{4,5}$, M. Beydler ${ }^{3}$, C.H. Chen ${ }^{6}$, P. Chen ${ }^{6}$, Y.C. Chen ${ }^{6}$, B.A. Clark ${ }^{7}$, W. Clay $^{8}$, A. Connolly ${ }^{1}$, L. Cremonesi ${ }^{9}$, P. Dasgupta ${ }^{10}$, J. Davies ${ }^{9}$, S. de Kockere ${ }^{11}$, K.D. de Vries ${ }^{11}$, C. Deaconu ${ }^{8}$, M. A. DuVernois ${ }^{3}$, J. Flaherty ${ }^{1}$, E. Friedman ${ }^{12}$, R. Gaior ${ }^{2}$, J. Hanson ${ }^{13}$, K. Hanson ${ }^{3}$, N. Harty ${ }^{14}$, B. Hendricks ${ }^{15,16}$, K.D. Hoffman ${ }^{12}$,


K.-C. $\mathrm{Kim}^{12}$, M.-C. Kim ${ }^{2}$, I. Kravchenko ${ }^{17}$, R. Krebs ${ }^{15,16}$, Y. Ku ${ }^{15,16}$, C.Y. Kuo ${ }^{6}$, K. Kurusu ${ }^{2}$, H. Landsman ${ }^{18}$, U.A. Latif ${ }^{4,11}$,

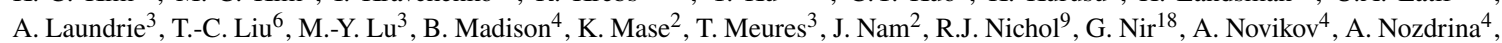
E. Oberla ${ }^{8}$, A. ÓMurchadha ${ }^{3}$, J. Osborn ${ }^{17}$, Y. Pan $^{14}$, C. Pfendner ${ }^{19}$, N. Punsuebsay ${ }^{14}$, J. Roth ${ }^{14}$, P. Sandstrom ${ }^{3}$, D. Seckel ${ }^{14}$, Y.S. Shiao ${ }^{6}$, A. Shultz ${ }^{4}$, D. Smith ${ }^{8}$, S. Toscano ${ }^{10}$, J. Torres ${ }^{1}$, J. Touart ${ }^{12}$, N. van Eijndhoven ${ }^{11}$, G.S. Varner ${ }^{20}$, A. Vieregg ${ }^{8}$, M.-Z. Wang ${ }^{6}$, S.-H. Wang 6 , Y.H. Wang ${ }^{6}$, S.A. Wissel ${ }^{15,16,21,22}$, S. Yoshida ${ }^{2}$, R. Young ${ }^{4}$

${ }^{1}$ Dept. of Physics, Center for Cosmology and AstroParticle Physics, The Ohio State University, Columbus, OH 43210

${ }^{2}$ Dept. of Physics, Chiba University, Chiba, Japan

${ }^{3}$ Dept. of Physics, University of Wisconsin-Madison, Madison, WI 53706

${ }^{4}$ Dept. of Physics and Astronomy, University of Kansas, Lawrence, KS 66045

${ }^{5}$ Moscow Engineering Physics Institute, Moscow, Russia

${ }^{6}$ Dept. of Physics, Grad. Inst. of Astrophys., Leung Center for Cosmology and Particle Astrophysics, National Taiwan University, Taipei, Taiwan

${ }^{7}$ Dept. of Physics and Astronomy, Michigan State University, East Lansing, Michigan 48824

${ }^{8}$ Dept. of Physics, Enrico Fermi Institue, Kavli Institute for Cosmological Physics, University of Chicago, Chicago, IL 60637

${ }^{9}$ Dept. of Physics and Astronomy, University College London, London, United Kingdom

${ }^{10}$ Université Libre de Bruxelles, Science Faculty CP230, B-1050 Brussels, Belgium

${ }^{11}$ Vrije Universiteit Brussel, Brussels, Belgium

${ }^{12}$ Dept. of Physics, University of Maryland, College Park, MD 20742

${ }^{13}$ Dept. Physics and Astronomy, Whittier College, Whittier, CA 90602

${ }^{14}$ Dept. of Physics, University of Delaware, Newark, DE 19716

${ }^{15}$ Center for Multi-Messenger Astrophysics, Institute for Gravitation and the Cosmos, Pennsylvania State University, University Park, PA 16802

${ }^{16}$ Dept. of Physics, Pennsylvania State University, University Park, PA 16802

${ }^{17}$ Dept. of Physics and Astronomy, University of Nebraska, Lincoln, Nebraska 68588

${ }^{18}$ Weizmann Institute of Science, Rehovot, Israel

${ }^{19}$ Dept. of Physics and Astronomy, Denison University, Granville, Ohio 43023

${ }^{20}$ Dept. of Physics and Astronomy, University of Hawaii, Manoa, HI 96822

${ }^{21}$ Dept. of Astronomy and Astrophysics, Pennsylvania State University, University Park, PA 16802

${ }^{22}$ Physics Dept., California Polytechnic State University, San Luis Obispo, CA 93407

\section{Acknowledgements}

We thank the National Science Foundation Office of Polar Programs and Physics Division for their generous support through NSF OPP902483, Grant NSF OPP-1002483, Grant NSF 1607555, Grant NSF OPP-1359535, Grant NSF OPP-1404212, Grant NSF OPP-2013134, and Grant NSF 2019597. 